\title{
Expansions of a chord diagram and alternating permutations
}

\author{
Tomoki Nakamigawa \\ Department of Information Science \\ Shonan Institute of Technology \\ Fujisawa, Kanagawa, Japan \\ nakami@info.shonan-it.ac.jp
}

Submitted: Mar 20, 2015; Accepted: Jan 4, 2016; Published: Jan 11, 2016

Mathematics Subject Classifications: 05A19, 05A05

\begin{abstract}
A chord diagram is a set of chords of a circle such that no pair of chords has a common endvertex. A chord diagram $E$ with $n$ chords is called an $n$-crossing if all chords of $E$ are mutually crossing. A chord diagram $E$ is called nonintersecting if $E$ contains no 2-crossing. For a chord diagram $E$ having a 2-crossing $S=\left\{x_{1} x_{3}, x_{2} x_{4}\right\}$, the expansion of $E$ with respect to $S$ is to replace $E$ with $E_{1}=(E \backslash S) \cup\left\{x_{2} x_{3}, x_{4} x_{1}\right\}$ or $E_{2}=(E \backslash S) \cup\left\{x_{1} x_{2}, x_{3} x_{4}\right\}$. It is shown that there is a one-to-one correspondence between the multiset of all nonintersecting chord diagrams generated from an $n$ crossing with a finite sequence of expansions and the set of alternating permutations of order $n+1$.
\end{abstract}

Keywords: chord diagram; alternating permutation; Entringer number; Euler number; Ptolemy's theorem

\section{Introduction}

Let us consider a set of chords of a circle. A set of chords is called a chord diagram, if they have no common endvertex. If a chord diagram consists of a set of $n$ mutually crossing chords, it is called an $n$-crossing. A 2 -crossing is simply called a crossing as well. If a chord diagram contains no crossing, it is called nonintersecting.

Let $V$ be a set of $2 n$ vertices on a circle, and let $E$ be a chord diagram of order $n$, where each chord has endvertices of $V$. We denote the family of all such chord diagrams by $\mathcal{C D}(V)$. Let $x_{1}, x_{2}, x_{3}, x_{4} \in V$ be placed on a circle in clockwise order. Let $E \in \mathcal{C D}(V)$. For a crossing $S=\left\{x_{1} x_{3}, x_{2} x_{4}\right\} \subset E$, let $S_{1}=\left\{x_{2} x_{3}, x_{4} x_{1}\right\}$, and $S_{2}=\left\{x_{1} x_{2}, x_{3} x_{4}\right\}$. The expansion of $E$ with respect to $S$ is defined as a replacement of $E$ with $E_{1}=(E \backslash S) \cup S_{1}$ or $E_{2}=(E \backslash S) \cup S_{2}$ (see Figure 1). In this procedure, $E$ is called the predecessor of $E_{1}$ 


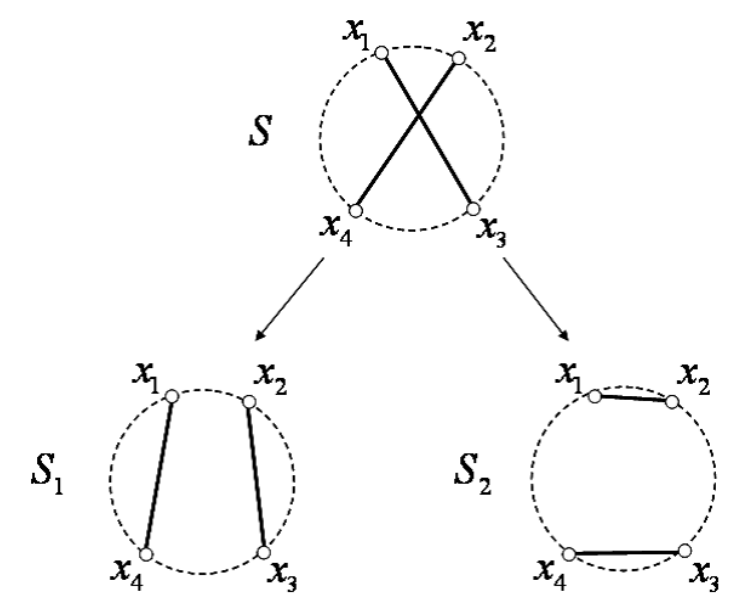

Figure 1: The expansion of a chord diagram.

and $E_{2}$, and $E_{1}$ and $E_{2}$ are called the successors of $E$. A chord of a chord diagram is called isolated, if it intersects no other chord.

For $E \in \mathcal{C D}(V)$, let us denote the number of 2-crossings of $E$ by $c(E)$. Let $E^{\prime}$ be a successor of $E$ such that $E^{\prime}=(E \backslash S) \cup S^{\prime}$, where $S$ is an original 2-crossing and $S^{\prime}$ is a pair of additional chords.

We claim that $c\left(E^{\prime}\right)<c(E)$. Indeed, for $e \in E \cap E^{\prime}$, let $t$ (resp. $t^{\prime}$ ) be the number of chords of $S$ (resp. $S^{\prime}$ ) intersecting $e$.

It is not difficult to see that if $t \leqslant 1$ then we have $t^{\prime}=t$, and if $t=2$ then we have $t^{\prime}=2$ or $t^{\prime}=0$. Hence, we have $t^{\prime} \leqslant t$. Since $S$ is a crossing of $E$ which is removed in $E^{\prime}$, we have $c\left(E^{\prime}\right)<c(E)$.

Lemma 1. Let $E \in \mathcal{C D}(V)$ be a chord diagram. Then beginning from $E$, the resulting mutiset of nonintersecting chord diagrams generated by a maximal set of expansions is uniquely determined.

Proof. We proceed by induction on the number of crossings $c$ of a chord diagram $E$.

If $c=0$ or 1 , there is nothing to prove. Let $c \geqslant 2$ and let $c(E)=c$. By inductive hypothesis, for a chord diagram $E^{\prime}$ with $c\left(E^{\prime}\right) \leqslant c-1$, we define $\mathcal{N C D}\left(E^{\prime}\right)$ as the resulting multisets of nonintersecting chord diagrams generated by $E^{\prime}$. Moreover, for a set of chord diagrams $\mathcal{E}$ such that $E^{\prime} \in \mathcal{E}$ with $c\left(E^{\prime}\right) \leqslant c-1$, let us denote $\mathcal{N C D}(\mathcal{E})=\cup_{E^{\prime} \in \mathcal{E}} \mathcal{N C D}\left(E^{\prime}\right)$.

Let $S_{1}$ and $S_{2}$ be two 2-crossings of $E$, and let $E_{i 1}$ and $E_{i 2}$ be two successors of $E$ by an expansion with respect to $S_{i}$ for $i=1,2$. Let $\mathcal{E}_{i}=\left\{E_{i 1}, E_{i 2}\right\}$ for $i=1,2$. What we want to show is that $\mathcal{N C D}\left(\mathcal{E}_{1}\right)=\mathcal{N C D}\left(\mathcal{E}_{2}\right)$.

Case 1. $S_{1} \cap S_{2}=\emptyset$.

For $E_{11}$ and $E_{12}$, by an expansion with respect to $S_{2}$, we have a set $\mathcal{E}^{\prime}$ of four chord diagrams. Then we have $\mathcal{N C D}\left(\mathcal{E}_{1}\right)=\mathcal{N C D}\left(\mathcal{E}^{\prime}\right)$. In the same way, for $E_{21}$ and $E_{22}$, by an expansion with respect to $S_{1}$, we have $\mathcal{E}^{\prime}$, and we have $\mathcal{N C D}\left(\mathcal{E}_{2}\right)=\mathcal{N} \mathcal{C D}\left(\mathcal{E}^{\prime}\right)$. Hence, we have $\mathcal{N C D}\left(\mathcal{E}_{1}\right)=\mathcal{N C D}\left(\mathcal{E}_{2}\right)$. 
Case 2. $S_{1} \cap S_{2} \neq \emptyset$.

We may assume $S_{1}=\left\{e_{0}, e_{1}\right\}$ and $S_{2}=\left\{e_{0}, e_{2}\right\}$, where $e_{i}=x_{i} y_{i}$ for $0 \leqslant i \leqslant 2$. Let $V_{0}=\left\{x_{0}, x_{1}, x_{2}, y_{0}, y_{1}, y_{2}\right\}$ and let $E^{\prime}=E \backslash\left\{e_{0}, e_{1}, e_{2}\right\}$. Beginning from $\mathcal{E}_{i}$ with $i=1,2$, let us consider expansions with respect to a crossing induced by $V_{0}$.

Case 2.1. $e_{1}$ and $e_{2}$ are not crossing.

We may assume $x_{0}, x_{1}, x_{2}, y_{0}, y_{2}, y_{1}$ are placed on a circle in clockwise order. By iterating possible expansions, not depending on the order of the expansions, we always have a set of four chord diagrams $\mathcal{E}^{\prime}=\left\{E^{\prime} \cup\left\{x_{0} x_{1}, x_{2} y_{0}, y_{2} y_{1}\right\}, E^{\prime} \cup\left\{x_{0} x_{1}, x_{2} y_{1}, y_{0} y_{2}\right\}\right.$, $\left.E^{\prime} \cup\left\{x_{0} y_{1}, x_{1} y_{2}, x_{2} y_{0}\right\}, E^{\prime} \cup\left\{x_{0} y_{1}, x_{1} x_{2}, y_{0} y_{2}\right\}\right\}$.

$\underline{\text { Case 2.2. }} e_{1}$ and $e_{2}$ are crossing.

We may assume $x_{0}, x_{1}, x_{2}, y_{0}, y_{1}, y_{2}$ are placed on a circle in clockwise order. By iterating possible expansions, not depending on the order of the expansions, we always have a set of five chord diagrams $\mathcal{E}^{\prime}=\left\{E^{\prime} \cup\left\{x_{0} x_{1}, x_{2} y_{0}, y_{1} y_{2}\right\}, E^{\prime} \cup\left\{x_{0} y_{2}, x_{1} x_{2}, y_{0} y_{1}\right\}\right.$, $\left.E^{\prime} \cup\left\{x_{0} x_{1}, x_{2} y_{2}, y_{0} y_{1}\right\}, E^{\prime} \cup\left\{x_{0} y_{0}, x_{1} x_{2}, y_{1} y_{2}\right\}, E^{\prime} \cup\left\{x_{0} y_{2}, x_{1} y_{1}, x_{2} y_{0}\right\}\right\}$.

In any case, we have $\mathcal{N C D}\left(\mathcal{E}_{i}\right)=\mathcal{N C D}\left(\mathcal{E}^{\prime}\right)$ for $i=1,2$, as required.

Let us denote the multiset of nonintersecting chord diagrams generated by $E \in \mathcal{C D}(V)$ by $\mathcal{N C D}(E)$. For $E \in \mathcal{C D}(V)$, let us define $f(E)$ as the cardinality of $\mathcal{N C D}(E)$ as a multiset.

Example 2. Let $C_{n}$ be an $n$-crossing. Then we have $f\left(C_{2}\right)=2, f\left(C_{3}\right)=5$ and $f\left(C_{4}\right)=16$. (See Figure 2.)

A background of expansions of a chord diagram is Ptolemy's theorem and its generalization. For two points $x, y$ on a circle, let $\overline{x y}$ be the length of a chord $x y$. Ptolemy's theorem states that if $E=\left\{x_{1} x_{3}, x_{2} x_{4}\right\}$ itself is a 2-crossing, then we have $\overline{x_{1} x_{3}} \cdot \overline{x_{2} x_{4}}=$ $\overline{x_{2} x_{3}} \cdot \overline{x_{4} x_{1}}+\overline{x_{1} x_{2}} \cdot \overline{x_{3} x_{4}}$. In other words, we have

$$
\prod_{e \in E} \bar{e}=\prod_{e \in E_{1}} \bar{e}+\prod_{e \in E_{2}} \bar{e}
$$

where $E_{1}$ and $E_{2}$ are two successors of $E$. In general, for a given $E \in \mathcal{C D}(V)$, by iterating expansions with applications of Ptolemy's theorem, we have

$$
\prod_{e \in E} \bar{e}=\sum_{E^{\prime} \in \mathcal{N C D}(E)} \prod_{e \in E^{\prime}} \bar{e}
$$

If $E$ is a 3 -crossing, the equation (2) is known as Fuhrmann's Theorem ([2]).

\section{Main Results}

For two nonnegative integers $k$ and $n$ with $k \leqslant n$, we define $A(n, k)$ as a chord diagram of order $n+1$, in which there is an $n$-crossing $E_{0}$ with an extra chord $e$ such that $e$ crosses 

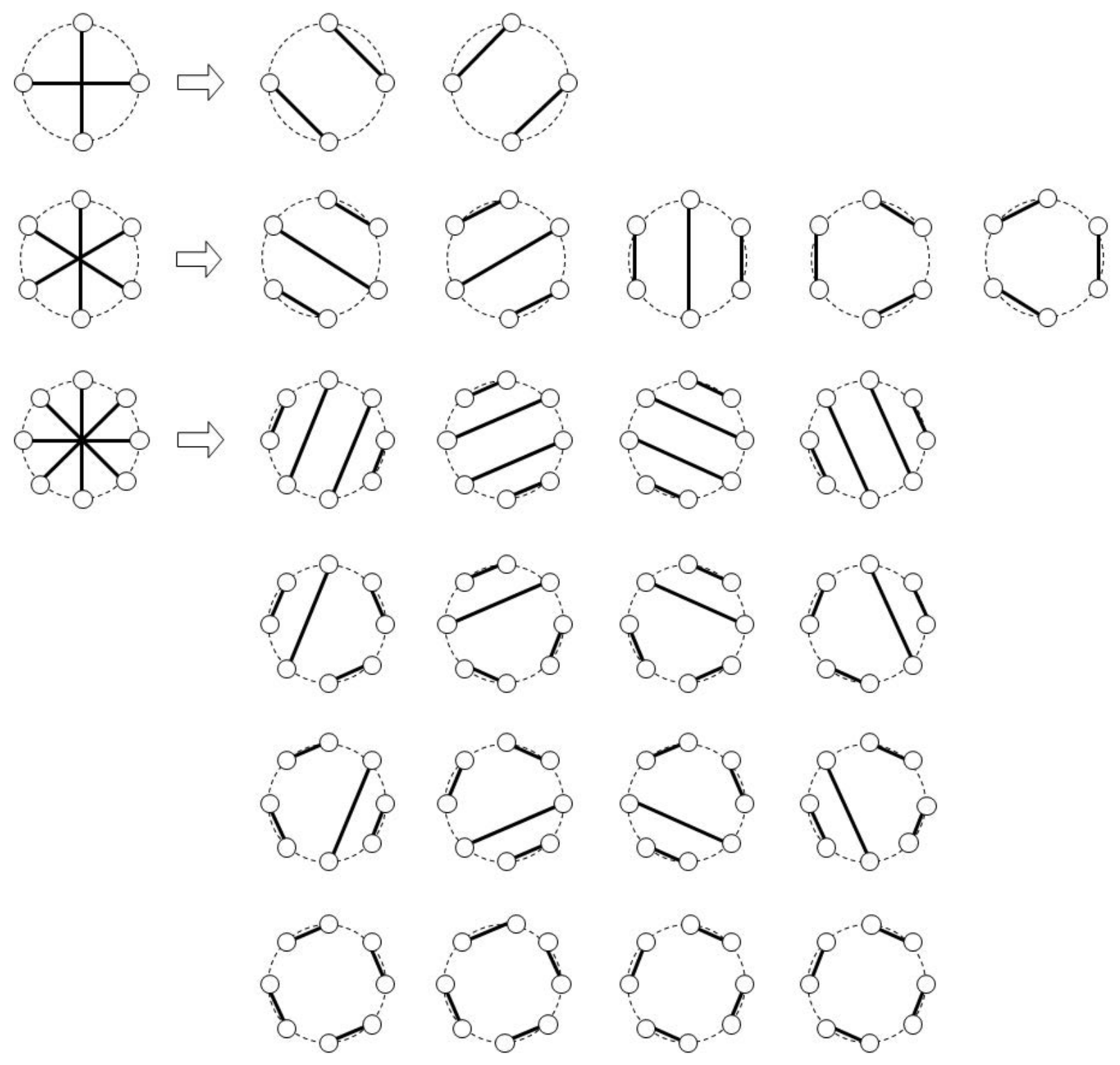

Figure 2: Multisets of nonintersecting chord diagrams generated by a 2-crossing(upper), a 3-crossing(middle) and a 4-crossing (lower). 
exactly $k$ chords of $E_{0}$. Note that $A(n-1, n-1)$ is simply an $n$-crossing, and that $A(n, 0)$ is a union of an $n$-crossing and an isolated chord. Hence, we have $f(A(n-1, n-1))=$ $f(A(n, 0))$. The values of $f(A(n, k))$ for small nonnegative integers $n$ and $k$ are shown in Table 1 .

Table 1: $f(A(n, k))$ for $0 \leqslant k \leqslant n \leqslant 6$.

\begin{tabular}{c|ccccccc}
$n \backslash k$ & 0 & 1 & 2 & 3 & 4 & 5 & 6 \\
\hline 0 & 1 & & & & & & \\
1 & 1 & 2 & & & & & \\
2 & 2 & 4 & 5 & & & & \\
3 & 5 & 10 & 14 & 16 & & & \\
4 & 16 & 32 & 46 & 56 & 61 & & \\
5 & 61 & 122 & 178 & 224 & 256 & 272 & \\
6 & 272 & 544 & 800 & 1024 & 1202 & 1324 & 1385
\end{tabular}

A permutation $\sigma$ of $[n]=\{1,2, \ldots, n\}$ is called an alternating permutation if $(\sigma(i)-$ $\sigma(i-1))(\sigma(i+1)-\sigma(i))<0$ for $2 \leqslant i \leqslant n-1$ (see [9] for an excellent survey of alternating permutations). An alternating permutation $\sigma$ is called an up-down permutation (resp. down-up permutation) if $\sigma(1)<\sigma(2)$ (resp. $\sigma(1)>\sigma(2))$. Let $\mathcal{U D} \mathcal{P}(n, k)$ denote the set of up-down permutations of $[n]$ with the first term at most $k$. Similarly, let $\mathcal{D U P}(n, k)$ denote the set of down-up permutations of $[n]$ with the first term at least $n-k+1$. Note that by definition, there is a natural bijection from $\mathcal{U D} \mathcal{P}(n, k)$ to $\mathcal{D U P}(n, k)$.

The main result of the paper is the following theorem.

Theorem 3. For $0 \leqslant k \leqslant n$, there is a bijection from $\mathcal{N C D}(A(n, k))$ to $\mathcal{U D P}(n+2, k+1)$.

For $0 \leqslant k \leqslant n$, Entringer number $E_{n, k}$ is defined as the number of down-up permutations of $[n+1]$ with the first term $k+1$ [1], which equals the cardinality of $\mathcal{U D P}(n, k)$. Since for $n \geqslant 1, E_{n+1,1}$ equals Euler number $E_{n}$, the number of down-up permutations of $[n]$, we have the following Corollary.

Corollary 4. For $0 \leqslant k \leqslant n$, we have $f(A(n, k))=E_{n+2, k+1}$. In particular, we have $f(A(n, 0))=E_{n+1}$.

Several combinatorial interpretations for Entringer numbers are known $([4,5,6,7,8])$. The generating function for Entringer number is treated in [3] as an exercise, Exer. 6.75. According to [3], it follows that

$$
\sum_{n \geqslant 0} \sum_{k \geqslant 0} E_{n+k, k} \frac{x^{n}}{n !} \frac{y^{k}}{k !}=\frac{\cos x+\sin y}{\cos (x+y)} .
$$

By Corollary 4, we have

$$
\begin{aligned}
\sum_{n \geqslant 0} \sum_{k \geqslant 0} f(A(n+k, k)) \frac{x^{n}}{n !} \frac{y^{k}}{k !} & =\frac{\partial^{2}}{\partial x \partial y}\left(\sum_{n \geqslant 0} \sum_{k \geqslant 0} E_{n+k, k} \frac{x^{n}}{n !} \frac{y^{k}}{k !}\right) \\
& =\frac{\cos x+\sin y}{\cos (x+y)(1-\sin (x+y))} .
\end{aligned}
$$




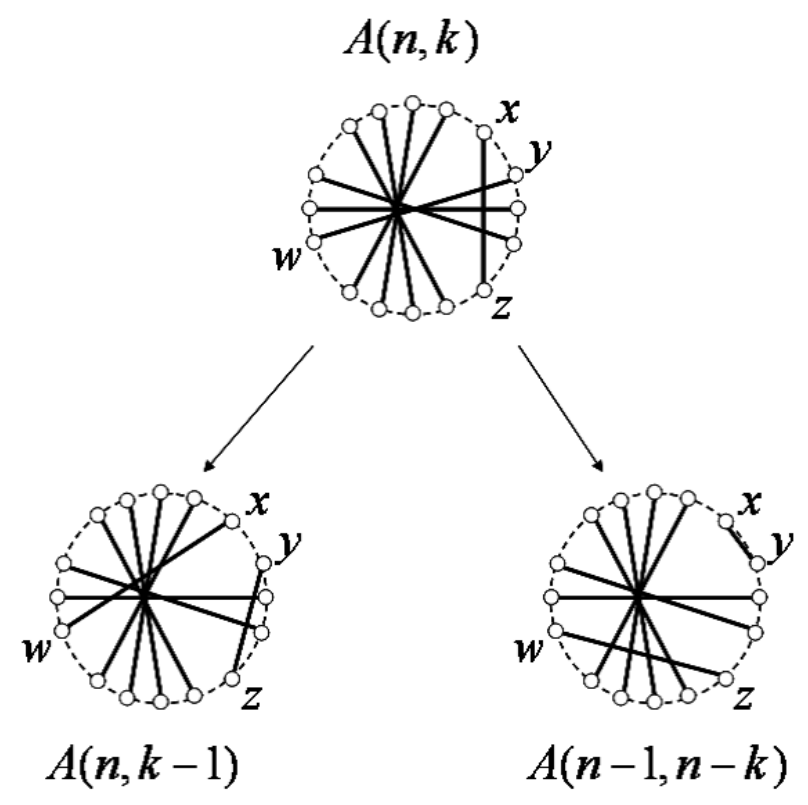

Figure 3: Two successors of $A(n, k)$, where $n=7$ and $k=3$.

\section{Proof of Theorem 3}

For two chord diagrams $F_{i} \in \mathcal{C D}\left(V_{i}\right)$ for $i=1,2$, let $F_{i}^{\prime} \in \mathcal{C D}\left(V_{i}^{\prime}\right)$ be a chord diagram such that $F_{i}^{\prime}$ consists of the set of all nonisolated chords of $F_{i}$. Suppose that $\left|F_{1}^{\prime}\right|=\left|F_{2}^{\prime}\right|$. Let $V_{i}^{\prime}=\left\{v_{i, 0}^{\prime}, v_{i, 1}^{\prime}, \ldots, v_{i, 2 n^{\prime}-1}\right\}$, and the vertices are placed on a circle in clockwise order for each $i=1,2$. Suppose that $v_{1, \alpha}^{\prime} v_{1, \beta}^{\prime} \in F_{1}^{\prime}$ holds if and only if $v_{2, \alpha}^{\prime} v_{2, \beta}^{\prime} \in F_{2}^{\prime}$ holds. Then we say that $F_{1}^{\prime}$ and $F_{2}^{\prime}$ are isomorphic, and furthermore we say that $F_{1}$ and $F_{2}$ are isomorphic as well.

In order to prove Theorem 3 , we will recursively construct a bijection from $\mathcal{N C D}(A(n, k))$ to $\mathcal{U D P}(n+2, k+1)$ for $0 \leqslant k \leqslant n$.

Firstly, we will show a recurrence for $\mathcal{N C D}(A(n, k))$, which is a key ingredient for the proof of Theorem 3 .

Lemma 5. For $1 \leqslant k \leqslant n$, we have a bijection between $\mathcal{N C D}(A(n, k))$ and $\mathcal{N C D}(A(n, k-$ $1)) \cup \mathcal{N C D}(A(n-1, n-k))$. In particular, we have $f(A(n, k))=f(A(n, k-1))+f(A(n-$ $1, n-k))$.

Proof. Let $E$ be a chord diagram isomorphic to $A(n, k)$. We may assume $E$ contains an $n$-crossing $E_{0}$ and an extra edge $e=x z$ such that $e$ crosses exactly $k$ edges of $E_{0}$.

Let $f=y w$ be an edge of $E_{0}$ such that (1) $x, y, z, w$ are placed on a circle in clockwise order and (2) there is no endvertex of $E_{0}$ between $x$ and $y$. (See Figure 3.)

Put $S=\{x z, y w\}$. Let us expand $E$ with respect to $S$. We have two successors $E_{1}, E_{2}$ of $E$, where $E_{1}=(E \backslash S) \cup\{y z, w x\}$ and $E_{2}=(E \backslash S) \cup\{x y, z w\}$. Then $E_{1}$ is isomorphic to $A(n, k-1)$ and $E_{2}$ is isomorphic to $A(n-1, n-k)$. Hence, we have a bijection between $\mathcal{N C D}(A(n, k))$ and $\mathcal{N C D}(A(n, k-1)) \cup \mathcal{N C D}(A(n-1, n-k))$. 
For the sake of completeness, we recall the well-known recurrence relation for $\mathcal{U D} \mathcal{P}(n, k)$. Lemma 6 . For $1 \leqslant k \leqslant n$, we have a bijection between $\mathcal{U D} \mathcal{P}(n+2, k+1)$ and $\mathcal{U D} \mathcal{P}(n+$ $2, k) \cup \mathcal{U D P}(n+1, n-k+1)$.

Proof. By the definition, $\mathcal{U D P}(n+2, k+1)$ is a set of up-down permutations of $[n+2]$ with the first term at most $k+1 \cdot \mathcal{U D} \mathcal{P}(n+2, k+1)$ is partitioned into $\mathcal{U D P}(n+2, k)$ and $\mathcal{T}=\mathcal{U D} \mathcal{P}(n+2, k+1) \backslash \mathcal{U D} \mathcal{P}(n+2, k)$, where $\mathcal{T}$ is a set of up-down permutations of $[n+2]$ with the first term $k+1$.

For $\sigma \in \mathcal{T}$, let us remove the first term of $\sigma$. The resulting permutation $\sigma^{\prime}$ is a downup permutation of $[n+2] \backslash\{k+1\}$ with the first term at least $k+2$. Hence, there is a natural bijection from $\mathcal{T}$ to $\mathcal{D U} \mathcal{P}(n+1, n-k+1)$, which has a one-to-one correspondence to $\mathcal{U D} \mathcal{P}(n+1, n-k+1)$.

Now, we return to the proof of Theorem 3.

For $n=0$ and $k=0$, a set of a single chord of $\mathcal{N C D}(A(0,0))$ clearly corresponds to a single permutation 12 of $\mathcal{U D P}(2,1)$.

Let $n \geqslant 1$ and $k \geqslant 0$. By the inductive hypothesis, we have a bijection from $\mathcal{N C D}\left(A\left(n^{\prime}, k^{\prime}\right)\right)$ to $\mathcal{U D P}\left(n^{\prime}+2, k^{\prime}+1\right)$ for $n^{\prime}<n$ or $n^{\prime}=n$ and $k^{\prime}<k$.

For $k=0, A(n, 0)$ is isomorphic to $A(n-1, n-1)$. Hence, there is a bijection from $\mathcal{N C D}(A(n, 0))$ to $\mathcal{N C D}(A(n-1, n-1))$. On the other hand, let $\sigma \in \mathcal{U D P}(n+2,1)$. By removing the first term of $\sigma$, we have a down-up permutation $\sigma^{\prime}$ of $[n+2] \backslash\{1\}$. Hence, there is a natural bijection from $\mathcal{U D} \mathcal{P}(n+2,1)$ to $\mathcal{D U P}(n+1, n)$, which has a one-to-one correspondence to $\mathcal{U D P}(n+1, n)$. Therefore, we have a bijection from $\mathcal{N C D}(A(n, 0))$ to $\mathcal{U D P}(n+2,1)$.

Let $k \geqslant 1$. In this case, by Lemma 5 and Lemma 6 , we can recursively construct a bijection from $\mathcal{N C D}(A(n, k))$ to $\mathcal{U D P}(n+2, k+1)$.

This completes the proof.

\section{Acknowledgments}

I thank an anonymous reviewer for his/her valuable suggestions. In particular, the proof of the main theorem was greatly simplified.

\section{References}

[1] R. C. Entringer, A combinatorial interpretation of the Euler and Bernoulli numbers, Nieuw. Arch. Wisk. 14(1966), 241-246.

[2] W. Fuhrmann, Synthetische Beweise Planimetrischer Sätze, Berlin, 1890.

[3] R. L. Graham, D. E. Knuth, and O. Patashnik, Concrete Mathematics, 2nd ed., Addison-Wesley, Reading, MA, 1994.

[4] Y. Gelineau, H. Shin, and J. Zeng, Bijections for Entringer families, Europ. J. Combinatorics, 32 (2011), 100-115.

[5] A. G. Kuznetsov, I. M. Pak, and A. E. Postnikov, Increasing trees and alternating permutations, Uspekhi Mat. Nauk, 49 (1994), 79-110. 
[6] J. Millar, N. J. A. Sloane, and N. E. Young, A new operation on sequences: the boustrophedon transform, J. Combinatorial Theory, Series A, 76 (1996), 44-54.

[7] C. Poupard, De nouvelles significations énumératives des nombres d'Entringer, Discrete Math., 38 (1982), 265-271.

[8] C. Poupard, Two other interpretations of the Entringer numbers, Europ. J. Combinatorics, 18 (1997), 939-943.

[9] R. P. Stanley, A survey of alternating permutations, Comtemporary Mathematics, 531 (2010), 165-196. 